\section{SP5-17 MONITORING ILLICIT DRUG USE AND SUBSTANCE DEPENDENCY AMONG FEMALE SEX WORKERS IN EUROPE, 2000-2009: AN OVERVIEW}

doi:10.1136/jech.2011.142976p.53

\section{Roelands. * Scientific Institute of Public Health, Brussels, Belgium}

Introduction Worldwide, illicit drug use and substance dependency will remain important public health problems during the next decades. Clinical evidence suggests that sex workers (SW) are a high risk group with increased prevalence. The study describes the state of affairs regarding monitoring illegal drug use among female sex workers in Europe and the existing gaps in our knowledge about the prevalence of use and dependency.

Methods Literature review: literature published between 2000 and 2009 was searched with PubMed. Moreover, the National Reports $(\mathrm{N}=222)$ provided yearly by the National Focal Points to the European Monitoring Centre on Drugs and Drug Addiction, were reviewed.

Results Prevalence estimates of illicit drug use in SW were found regarding 16 countries. Levels of illicit drug use and substance dependency were found to be high compared to the general population and differed by geographical area, age and working environment. Available data suggest a trend towards increasing illicit drug use among SW in Europe.

Conclusion There is substantial evidence that prevalence of illicit drug use and substance dependency are considerable higher in SW. However, because most European countries lack surveys and surveillance systems that provide solid estimates of drug use in this vulnerable population, additional research is needed. Feasibility of monitoring drug use by population-specific health services should be studied as this approach would facilitate timely interventions.

\section{SP5-18 INFLUENCE OF GENDER ON POSITIVE MENTAL HEALTH OF YOUNG PEOPLE IN KERALA}

doi:10.1136/jech.2011.142976p.54

S Mathew, ${ }^{*}$ V Raman Kutty. Sree Chitra Tirunal Institute for Medical Sciences and Technology, Trivandrum, Kerala, India

Introduction We examined gender differences in the positive mental health $(\mathrm{PMH})$ status, which encompasses positive states of minds and positive functioning, among young people of Kerala state, India.

Methods Using a scale development and validation process with 323 randomly selected subjects, we constructed and validated a scale for measuring PMH. This was administered to the study sample (453; 230 males, 223 females) in the age group of 18-24.

Results Males scored higher than females in the scale (males, $\mathrm{M}=64.878, \mathrm{SD}=10.695$, and females $\mathrm{M}=58.26, \mathrm{SD}=13.80 ; \mathrm{t}(451)=$ $5.70, \mathrm{p} \leq 0.01)$. Of the four domains of $\mathrm{PMH}$, three showed significantly higher scores in males (own potential: male- $M=21.34$, $\mathrm{SD}=5.38$; female- $\mathrm{M}=19.848, \mathrm{SD}=5.784$; coping abilities: male$\mathrm{M}=13.130, \mathrm{SD}=4.274$; female- $\mathrm{M}=10.910, \mathrm{SD}=3.969$; productivity: male- $\mathrm{M}=15.830, \mathrm{SD}=4.009$; female $=13.475, \mathrm{SD}=5.838$ ). Among subjects in the highest quartile of the score, $62.8 \%$ are males and $37.2 \%$ are females, out of a total of $113(24.9 \%$ of the total population).

Conclusion We found a perceptibly higher PMH score for men compared to women in this population. The role that parenting (perceived parenting style, gender preferences of the parents and single parenting) and traditional role stereotypes play, may be the strong factors deciding this difference in the scores.

\section{SP5-19 WHAT IS THE ROLE OF OCCUPATIONAL TRANSMISSION IN THE EPIDEMIOLOGY OF HELICOBACTER PYLORI?}

doi:10.1136/jech.2011.142976p.55

${ }^{1,2} \mathrm{~A}$ De Schryver, ${ }^{*} \mathrm{M}$ Van Winckel, ${ }^{2} \mathrm{~W}$ Van Hooste, ${ }^{2} \mathrm{G}$ Wullepit, ${ }^{2} \mathrm{~K}$ Cornelis, ${ }^{2} \mathrm{~A} M$ Charlier, ${ }^{2} \mathrm{~K}$ Colemonts. ${ }^{1}$ University of Antwerp, Epidemiology and Social Medicine, Antwerp, Belgium; ${ }^{2}$ IDEWE Occupational Health Services, Leuven, Belgium ${ }^{3}$ Clinic of Paediatrics, Ghent University, Ghent, Belgium

Introduction One of the surprising features of $H$ pylori, is that we still do not know how the organism is usually acquired. Two possible avenues for transmission are direct infection from person to person, or through an intermediary such as the water supply, flies or animals. If transmission is person to person, the organism may be transmitted by faeco-oral, oro-oral, or by gastro-oral route.

Methods In order to study some of these aspects we performed a number of cross-sectional studies, the results of which are reported here.

\section{Results}

Faeco-oral transmission: In a cross-sectional study in 587 employees working in 2 institutions for children with mental disabilities with a documented high prevalence of $H$ pylori infection, using multiple regression analysis to adjust for confounding variables, we found an almost 2.5 increased risk in workers having contact with faeces of inhabitants. Other risk factors for $H$ pylori infection were not significant in multiple regression analysis. In another cross-sectional study in 198 nursing home workers, the ageadjusted prevalence of $H$ pylori was not higher than in a reference population and no association with any other risk factors was found.

Waterborne transmission: In a cross-sectional study in 317 wastewater workers, the age-adjusted prevalence of $H$ pylori was not higher than in a reference population and there was no association with any activity by which workers were exposed to wastewater. Conclusions Results of our studies show the difficulty in interpreting cross-sectional studies. There is a need for cohort studies with an exact exposure assessment to all possible transmission routes.

\section{SP5-20 SOCIAL STIGMA AS A DETERMINANT FOR LEPROSY ELIMINATION IN CAMEROON}

doi:10.1136/jech.2011.142976p.56

D S Nsagha, ${ }^{*}$ S M Nsagha, E Tabah, A Njunda, J C Assob, E A Bamgboye, A Oyediran, P N Fon, A K Njamnshi. University of Buea, Buea, South West Region, Cameroon

Objective To investigate the role of social stigma as a determinant for leprosy elimination in a leprosy endemic region of Cameroon.

Methods Focus group discussions, in-depth interviews and structured questionnaires were used to investigate leprosy social stigma among lepers, their contacts and a control group consisting of patients attending a health facility for reasons other than leprosy. Informed consent was sought and gained before the commencement of the study.

Results Focus group discussions and indepth interviews identified three types of stigma: lack of self-esteem, tribal stigma and complete rejection by society. From the 480 structured questionnaires administered, there were overall positive attitudes to lepers among the study population and within the divisions $(p=0.0)$. The proportion of participants that felt sympathetic with deformed lepers was $78.1 \%$ (95\% CI $74.4 \%$ to $81.8 \%$ ) from a total of 480.399 (83.1\%) respondents indicated that they could share a meal or drink at the same table with a deformed leper (95\% CI 79.7\% to $86.5 \%$ ). $403(83.9 \%$ ) [95\% CI $80.7 \%$ to $87.3 \%$ ] participants indicated that they could have a handshake and embrace a deformed leper. A total 